\title{
OCUPACIONES HUMANAS TARDÍAS EN LA TRANSICIÓN BOSQUE ESTEPA: LA LOCALIDAD DE WINCHESTER (CURSO ALTO DEL RÍO CISNES, REGIÓN DE AISÉN).
}

\author{
OMAR REYES B." "“", CÉSAR MÉNDEZ M."*", HÉCTOR VELÁSQUEZ M."* Y VALENTINA TREJO V.
}

\begin{abstract}
RESUMEN
Se presentan nuevos antecedentes sobre los últimos momentos de la ocupación del valle del río Cisnes por parte de grupos cazadores recolectores de la estepa. Los resultados corresponden a nuevos hallazgos en la transición bosque estepa, particularmente referidos al sitio Winchester 1 (CIS 074). Se comenta su importancia y similitud respecto a otros registros ubicados en la estepa extra andina, como el sitio Appeleg 1(CIS 009). Finalmente, se discute su cronología en relación a las ocupaciones de distintos espacios/ambientes del valle durante el Holoceno tardío.
\end{abstract}

PALABRAS CLAVES: Cazadores recolectores, transición bosque estepa, Holoceno tardío.

\section{LATE HUMAN OCCUPATIONS IN THE FOREST/STEPPE TRANSITION: WINCHESTER LOCALITY (UPPER CISNES RIVER, XI REGIÓN DE AISÉN)}

\begin{abstract}
We present new information regarding the final moments of steppe hunter-gatherers' occupation of Cisnes River basin. Results are new findings in the forest/steppe transition, particularly Winchester 1 site (CIS 074). We comment its importance and similarity to other steppe extra Andean records, like Appeleg 1 site (CIS 009). Finally, we discuss its chronology in relation to the occupation of different spaces/environments of the valley during the late Holocene.
\end{abstract}

KEY WORDS: Hunter-gatherers, forest/steppe transition, late Holocene.

\section{ANTECEDENTES}

La investigación arqueológica del valle del río Cisnes $\sim 44^{\circ}$ S) ha permitido el descubrimiento $y$ registro de sitios en todos sus segmentos ambien- tales. Éstos han sido caracterizados en relación a su distribución y componentes, e integrados en explicaciones relativas a la movilidad y el asentamiento (Méndez et al. 2007, Reyes et al. 2006). Los resultados han permitido la reevaluación del tema

* Centro de Estudios del Hombre Austral, Instituto de la Patagonia, Universidad de Magallanes. Bulnes 1890, Punta Arenas.

* Centro de Estudios Humanos y Patrimoniales (CEHP). Nataniel Cox 31. Of. 57. Santiago.

** Departamento de Antropología, Facultad de Ciencias Sociales, Universidad de Chile. Ignacio Carrera Pinto 1045, Nuñoa, Santiago. 
de las incursiones de cazadores recolectores de las estepas en ambientes considerados tradicionalmente como "marginales", como son las áreas boscosas del oeste de Patagonia (Mena 1995, Bate y Mena 2005, Méndez et al. 2006). Las discretas ocupaciones detectadas en estos ambientes montano-boscosos se encuentran claramente documentadas hacia los 2800 a 2300 años calendarios AP, momento en donde se identifica una conectividad a lo largo del curso fluvial (Méndez y Reyes 2006, Reyes et al. 2007a), mientras que el ambiente estepario fue utilizado desde tiempos finipleistocenos (Reyes et al. 2007b). A raíz de lo anterior, detectadas las ocupaciones, una de las preguntas más interesantes resultaba ser, qué tanto se prolongó en el tiempo la incorporación de los ambientes al oeste de la estepa del río Cisnes a los circuitos de movilidad. Los resultados que presentamos a continuación, corresponden a datos preliminares que pretenden aportar a la comprensión de las dinámicas de ocupación de los espacios de transición bosque estepa. Esta singular área intercala, por un lado, elementos vegetales propios de la estepa arbustiva con bosques caducifolios en galería, y por otro, relieves accidentados como los cordones subandinos orientales con valles bajos y el inicio de los relieves planiformes (Guajardo 1994, SERPLAC 2005). Presenta, por tanto, particulares desafíos, tanto para la ocupación humana pretérita, como para la detección de los hallazgos que dan cuenta de ella.

A continuación, describimos algunos contextos y conjuntos arqueológicos registrados en el ambiente de transición bosque-estepa del río Cisnes, durante las campañas de terreno de 2005 y 2007. Los resultados se limitan a información superficial, tanto de prospección, como de recolecciones intensivas. Las similitudes entre algunos atributos observados en los contextos con sitios análogos identificados en la estepa aledaña se destacan como los elementos más significativos. La cronología obtenida en uno de estos sitios permite posicionar de forma relativa los hallazgos de la localidad en relación a las ocupaciones del valle en general.

\section{CONTEXTOS ARQUEOLÓGICOS EN LA TRANSICIÓN BOSQUE ESTEPA}

Se llevaron a cabo prospecciones pedestres en el área donde se puede identificar actualmente la transición entre el bosque y la estepa. El área muestreada cubre un total de $18 \mathrm{~km}^{2}$. Se divide en tres sectores, a saber: Winchester $\left(\sim 6 \mathrm{~km}^{2}\right)$ en la margen sur del río Cisnes (Fig. 1.1), el sector Loma Chica $\left(\sim 4 \mathrm{~km}^{2}\right)$ en la margen norte del mismo curso (Fig. 1.2) y río Mallín Chileno $\left(\sim 8 \mathrm{~km}^{2}\right)$ al norte de la hoya hidrográfica del Cisnes (Fig. 1.3).

El sector de río Mallín Chileno corresponde a un amplio y alto valle limítrofe $(900 \mathrm{msnm}$ en promedio) que corre en dirección NE-SO, detrás los cordones cordilleranos Cáceres y Carlota (por el S) y la cordillera Steffen (por el N) para finalmente desembocar en el poblado de La Tapera por el curso fluvial Cáceres. Se caracteriza por grandes bosques de hoja caduca, intercalados por extensos campos de morrenas con vegetación de estepa arbustiva. Además, se observan extensas zonas de mallines y pantanos permanentes que dificultan, tanto la accesibilidad, como la visibilidad del lugar. Cabe agregar finalmente, que en esta área no se registraron elementos arqueológicos. No obstante, queda de manifiesto la gran diversidad de ambientes que estos cursos fluviales ofrecen de acuerdo a su orientación y altitud. En efecto, se trata de un valle muy húmedo, por lo que presenta una cubierta boscosa mayor respecto a la situación observada en el curso del Cisnes (misma longitud y distante $12 \mathrm{~km}$ más al sur), habiendo afectado la cobertura de prospección.

Loma Chica ( 650 msnm) corresponde a un sector de transición donde el bosque caducifolio y la estepa arbustiva se intercalan, esta última, domina particularmente en las partes abiertas. El relieve puede ser caracterizado como planiforme con sinuosidades leves de tipo lomaje. En esta área se identificaron, sobre unas morrenas glaciares, una serie de acumulaciones intencionales de piedras en estado disturbado por agentes antrópicos (Reyes et al. 2006). Adicionalmente se recuperó un desecho de talla lítica aislado. Los sitios fueron identificados con siglas de CIS 030, 031, 032 y 033.

Por otro lado, en la margen sur del río Cisnes, fue posible recorrer mayor cantidad de sectores planos y quebradas situados entre los arroyos Winchester y Winchester Sur. En este sector se registraron dos sitios caracterizados por una gran diversidad de material arqueológico, sin intrusiones históricas (p.e. metales, vidrios). 

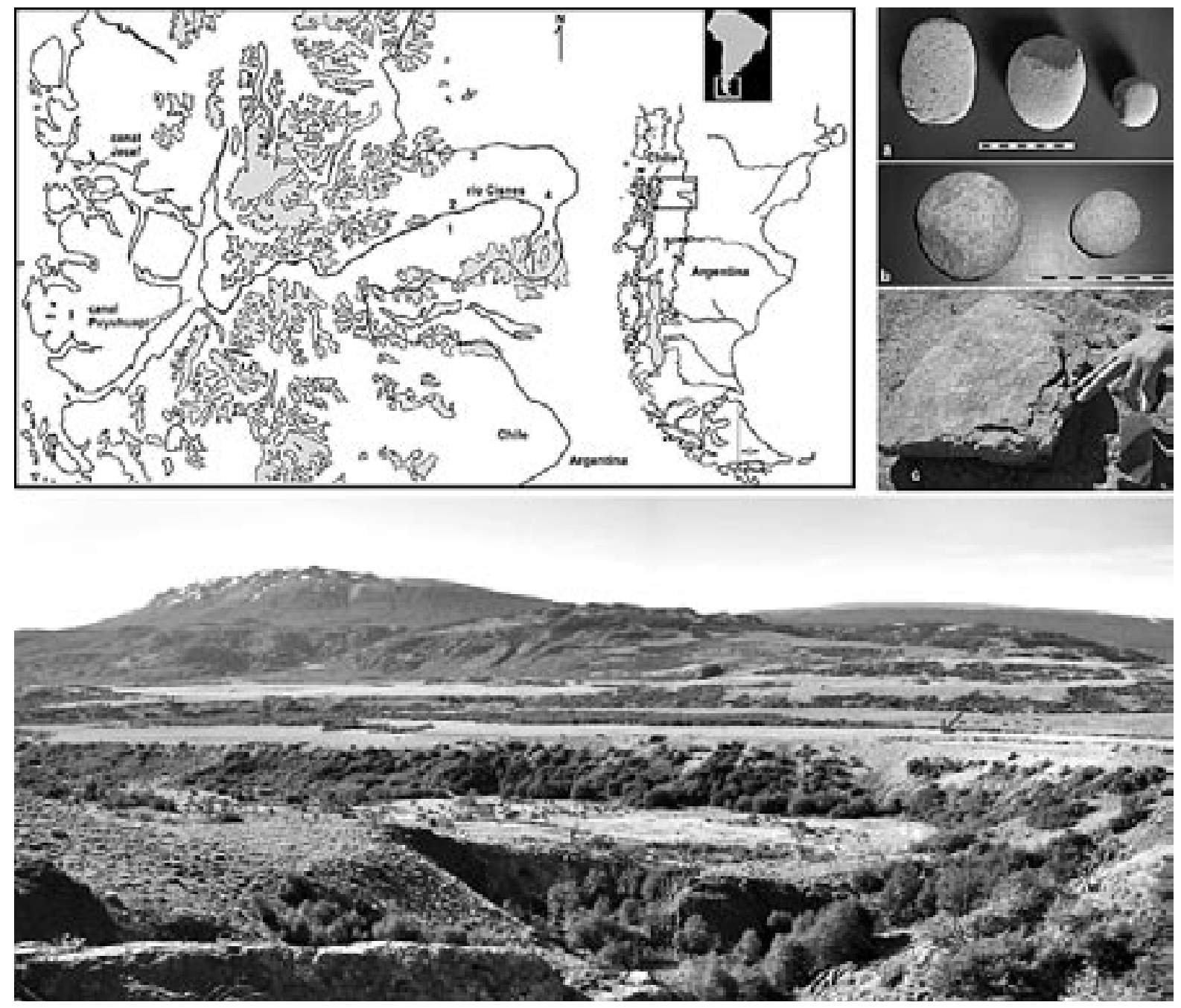

Fig. 1. Ubicación de los sitios y localidades referidas en el texto, 1. Winchester, 2. Loma Chica, 3. río Mallín Chileno, 4. Appeleg, 5. Alero Las Quemas. Materiales recuperados, a. implementos de molienda, b. bolas en distintos estadios de producción, c. laja/soporte con pulimento y canteo marginal; escalas: $10 \mathrm{~cm}$. Abajo: ambiente y emplazamiento de Winchester 1 desde Winchester 2, mostrando cima de morrena sobre la que se dispone el sitio.

El sitio Winchester 1 (CIS 074, 660 msnm) se ubica sobre una morrena incidida por el curso de Winchester Sur. Éste define un perfil abrupto entre la cima de la morrena (área del sitio) y la parte baja del valle. El sitio corresponde a una dispersión de materiales que cubre $\sim 3500 \mathrm{~m}^{2}$. Los materiales se disponen sobre una duna arenosa de poco espesor sobre la morrena erosionada. La vegetación en el área del sitio es limitada, aunque en las inmediaciones corresponde a taxones arbustivos, propios de la estepa. Por su parte, en los fondos de valle se observan discretos bosques de Nothofagus en galería. Entre los materiales en superficie dominan ampliamente los líticos, aunque fue posible identificar pocos huesos en superficie. Entre estos últimos identificamos un metapodio de guanaco (Lama guanicoe), y otros huesos largos de mamíferos y aves. Particularmente los restos óseos de mamíferos se encuentran en significativo estadio de meteorización (5 de acuerdo a Behrensmeyer 1978) por el efecto de exposición. Estas evidencias no se consideran necesariamente como sincrónicas a los hallazgos líticos y bien podrían responder a incorporaciones tafonómicas. 
Los materiales líticos se observaron tanto en concentraciones, como de forma dispersa. Pese a su naturaleza superficial -que sugiere que las asociaciones no son necesariamente un reflejo sistémico- algunas categorías como raspadores, sobadores para el tratamiento de cuero y otros instrumentos, fueron registradas cerca de concentraciones discretas de rocas quemadas que conformaban emplantillados de fogones semienterrados. Estas áreas de concentración totalizan tres, de las cuales una fue muestreada para su datación por termoluminiscencia. Adicionalmente, también en asociación a los emplantillados, se registró una laja/soporte con una superficie ligeramente pulida y un borde canteado (Fig 1.c), probablemente para la realización de actividades que requirieran apoyo.

Entre las categorías líticas identificadas se observó cuchillos bifaciales, raspadores frontales sobre lascas y sobre láminas, pequeñas láminas con retoque marginal, manos de moler formatizadas e informales (Fig. 1.a) y bolas líticas. Los instrumentos formatizados sobre lascas y láminas se encuentran manufacturados sobre finos sílices en distintos grados de traslucidez. Aún así la mayoría de la muestra se compone de rocas de grano más grueso, como andesitas, representadas principalmente por derivados de núcleo. Desechos de talla marginal y bifacial, son muy minoritarios, aunque en rocas de mayor calidad. Una parte importante del conjunto se compone por instrumentos sobre guijarros. Éstos, mediante piqueteo y pulimento, fueron transformados en manos de moler $(n=3)$ y bolas líticas $(n=2)$. Estas últimas se observan en distintos estados de producción (Fig. 1.b). Lo más probable es que las materias primas para estas categorías fueran obtenidas de las inmediaciones, correspondiendo incluso a las mismas rocas de la morrena. Finalmente, se suma un componente de instrumentos informales tallados, entre lo que se incluye cepillos sobre guijarros -como tajadores (corte por percusión)- y toscos instrumentos sobre núcleo, seguramente implementados en el aprovechamiento de recursos de madera.

En atención a las categorías artefactuales identificadas y los rasgos contextuales en superficie y reciente exposición se sugiere que la mayoría de las actividades llevadas a cabo en el asentamiento fueron de índole domésticas. Ello permite suponer que Winchester 1 corresponda a un campamento residencial emplazado en la inmediatez del un curso fluvial menor, donde probablemente se congregaron variados recursos.

Por su parte, el sitio Winchester 2 (CIS 075, $710 \mathrm{msnm}$ ), se emplaza sobre una suave ladera de exposición norte, en un área más restringida de $\sim 200 \mathrm{~m}^{2}$, y a $300 \mathrm{~m}$ más al sur de Winchester 1 . A diferencia de este último, la vegetación circundante corresponde a un bosque caducifolio achaparrado con intervalos de estepa arbustiva. La cubierta vegetacional, intercala coirones con amplios arenales donde es posible observar desechos de talla lítica esparcidos y en muy baja densidad. Posiblemente la menor cuantía de materiales se deba a la potencia mayor de la cubierta o bien a diferencias funcionales respecto a CIS 074.

\section{CRONOLOGÍA Y OCUPACIÓN TARDÍA DE LA TRANSICIÓN BOSQUE ESTEPA}

Ambos asentamientos mencionados, especialmente Winchester 1 (CIS 074), presentan características tecnológicas, tipológicas, funcionales y recursos líticos similares a los sitios Appeleg 1 y 2 (CIS 009 y CIS 018, Méndez et al. 2007), distante $20 \mathrm{~km}$ hacia el $\mathrm{E}$, en la estepa aledaña (Velásquez et al. 2007). Aún cuando difieren en el tipo de emplazamiento, extensión de los sitios, presencia de materiales cerámicos e históricos y ubicación en ambiente de estepa arbustiva versus estepa transicional, observamos ciertas afinidades y relaciones entre ambos. Así, materiales tipológicamente asignables al Holoceno tardío (p.e. bolas líticas, laminas retocadas, raspadores frontales, manos de moler), además de la presencia de emplantillados para fogones y soportes para actividades, documentados en la estepa (Velásquez et al. 2007), permitía establecer "relaciones" entre los campamentos esteparios situados al oriente y los sitios ubicados en la transición, hacia el occidente del valle.

Dada la ausencia de estratigrafía y elementos orgánicos en Winchester 1 , se seleccionó una roca tiznada-proveniente de un emplantillado- para su datación por termoluminiscencia. La asignación temporal de $790 \pm 80$ años (UCTL 1870), permitió situar contemporáneamente esta ocupación con alguno de los múltiples eventos del campamento Appeleg 1 , entre los $\sim 1290$ (sobre roca de emplantillado de fogón) y 740 años (sobre un fragmento de cerámi- 
ca) con ayuda del mismo método (Velásquez et al. 2007). La contemporaneidad y afinidades culturales permitían suponer que estos sitios en la transición bosque estepa estarían integrados a los rangos de circulación de materiales y personas.

Si bien se ha documentado rangos de movilidad valle adentro en los bosques montanos del río Cisnes hacia los 2800 a 2300 años cal. AP, éstos parecen no haber persistido más allá de este tiempo. Los resultados presentados permiten proponer que con posterioridad a dicho lapso, los grupos tardíos limitaron sus rangos de acción a la estepa y pisos bajos de la transición bosque-estepa. Resulta interesante esta reconfiguración de la movilidad en atención a las complejas situaciones ambientales y sociales sugeridas contemporáneamente para el interior de Patagonia Centro Meridional (Goñi y Barrientos 2000, Goñi et al. 2004, Mena y Lucero 2004). Aún así, es fundamental entender los avances y retrocesos del bosque y la estepa de acuerdo a las pulsaciones climáticas post pleistocénicas, las cuales han sido caracterizadas como muy variables posterior a los últimos 5000 años (Markgraf et al. 2003).

\section{AGRADECIMIENTOS}

Esta investigación fue financiada por CONICYT a través de su proyecto FONDECYT 1050139. Se agradece también el apoyo irrestricto de Estancia Río Cisnes para efectuar nuestra labor, a los arqueólogos Claudia Quemada, Carolina Belmar y Daniel Quiroz, a nuestros colaboradores de Villa La Tapera, Andrés Hernández y de Mañihuales, Leonardo Zúñiga. Finalmente, nuestra gratitud a Carabineros de Chile del puesto fronterizo Frías-Appeleg por su incondicional y permanente ayuda.

\section{BIBLIOGRAFÍA}

BATE, F. y F. MENA. 2005. Alero El Toro: un campamento indígena en el bosque siempreverde cercano al litoral en Aisén. En: Actas del XVI Congreso Nacional de Arqueología Chilena: 675-680. Museo de Historia Natural de Concepción y Sociedad Chilena de Arqueología.

BEHRENSMEYER, A. 1978. Taphonomic and ecologic information from bone weathering. Paleobiology 4(2): 150-162.

GUAJARDO, R. 1994. La vegetación natural de Chile. Editorial Universitaria, Santiago.
GOÑI, R. y G. BARRIENTOS. 2000. Estudio de chenques en Lago Salitroso, Provincia de Santa Cruz. En: Desde el país de los gigantes. Perspectivas arqueológicas en Patagonia, Tomo I:161-155. Universidad de Nacional de la Patagonia Austral, Río Gallegos.

GOÑI, R., G. BARRIENTOS, M. FIGUERERO, G. MENGONI, F. MENA, V. LUCERO y O. REYES. 2004. Distribución espacial de entierros en la cordillera de Patagonia centromeridional (Lago Salitroso-Paso Roballos, Argentina. Entrada Baker-Chacabuco, Chile). En: Actas XV Congreso Nacional de Arqueología Chilena. Chungara Revista de Antropología Chilena volumen especial, tomo II: 1101-1107.

MARKGRAF, V., J. PLATT BRADBURY, A. SCHWALB, S. BURNS, C. STERN, D. ARIZTEGUI, A. GILLI, F. ANSELMETTI, S. STINE y N. MAIDANA. 2003. Holocene palaeoclimates of southern Patagonia: limnological and environmental history of Lago Cardiel, Argentina. The Holocene 13(4):581-591.

MENA, F. 1995. El ser humano y su larga relación con el bosque. Ambiente y Desarrollo XI(1): 63-69.

MENA, F. y V. LUCERO. 2004. En torno a las últimas poblaciones indígenas de la cordillera centro-patagónica: estudio comparado de tres valles en Aisén oriental (Chile). En: Contra viento y marea. Arqueología de Patagonia, editado por M. Civalero, P. Fernández y G. Guráieb, pp. 643-657. Instituto Nacional de Antropología y Pensamiento Latinoamericano, Buenos Aires.

MÉNDEZ, C. y O. REYES. 2006. Nuevos datos de la ocupación humana en la transición bosque estepa en Patagonia: alero Las Quemas (Comuna de Lago Verde, XI Región de Aisén). Magallania 34(1):161-165.

MÉNDEZ, C., O. REYES y H. VELÁSQUEZ. 2007. Tecnología lítica en el alto río Cisnes (estepa extra andina de la XI Región de Aisén): primeros resultados. Boletín de la Sociedad Chilena de Arqueología 39. En prensa.

MÉNDEZ, C., H. VELÁSQUEZ, O. REYES y V. TREJO. 2006. Tras los moradores del bosque. Análisis de los conjuntos arqueológicos de Alero El Toro (Valle del río Cisnes, Región de Aisén). Werken 8: 101-115.

REYES, O., C. MÉNDEZ y H. VELÁSQUEZ. 2007a. De las estepas a los canales septentrionales: Uso humano de distintos ambientes/bosques del valle del río Cisnes (Holoceno tardío, XI Región de Aisén). En: Actas del XVII Congreso Nacional de Arqueología Chilena. Universidad Austral, Valdivia. En prensa.

REYES, O., C. MÉNDEZ, H., V. TREJO y H. VELÁSQUEZ. 2007b. El Chueco 1: un asentamiento multicomponente 
en la estepa occidental de Patagonia Central (11400 a 2700 años cal ap., $44^{\circ}$ s) Magallania 35(1):61-74. REYES, O., C. MÉNDEZ, H. VELÁSQUEZ y V. TREJO. 2006. Distribuciones espaciales y contextos arqueológicos de cazadores recolectores esteparios en Alto río Cisnes (XI Región de Aisén). Magallania 34(2):75-90.

SERPLAC XI REGION. 2005. Atlas Región de Aisén. LOM Ediciones, Santiago.
VELÁSQUEZ, H., C. MÉNDEZ, O. REYES, V. TREJO, L. SANHUEZA, D. QUIROZ y D. JACKSON. 2007 Campamentos residenciales tardíos a cielo abierto en el alto rio Cisnes (XI Región de Aisén): Appeleg 1 (CIS 009) Magallania 35(1):85-98. 